\title{
Diversity Order Results for MIMO Optical Wireless Communications
}

\author{
Yerzhan Sapenov, Anas Chaaban, Zouheir Rezki, Mohamed Abdallah, Khalid Qaraqe and Mohamed-Slim Alouini
}

\begin{abstract}
An optical wireless multiple-input multiple-output (MIMO) communication system employing intensity-modulation direct-detection (IM/DD) is considered. The maximal diversity order of the channel is characterized by studying the outage probability. Then, spatial repetition coding $(\mathrm{RC})$ is shown to be diversity-optimal as it achieves the channel's maximal diversity order. This diversity order is given by a simple expression which is suitable for any channel statistics of practical interest. The results are specialized to some practical channel statistics, and numerical results are provided to verify the results.
\end{abstract}

Index Terms-Intensity-modulation direct-detection, optical wireless communications, repetition coding, diversity order.

\section{INTRODUCTION}

Optical wireless communication (OWC) offers an attractive alternative to current radio frequency (RF) systems for achieving high data rates and contributing to the evolution of wireless networks. This made OWC an attractive research topic for both industry and academia [1].

Different aspects of OWC have been studied in the literature. Of particular interest is the simple and relatively cheap implementation of OWC using IM/DD, whose performance has been studied extensively [2]-[4]. This letter focuses on MIMO OWC, which has been recently studied from error probability perspective [2], and capacity perspective [3], [4]. Since outdoor OWC systems are prone to channel variations due to atmospheric turbulence and pointing errors [5], the optimal diversity order of these systems is an important performance criterion for reliable communications.

Thus, we consider a MIMO IM/DD system, subject to per-aperture optical intensity constraints, which are common and realistic constraints in IM/DD operation. Recently, [4], [6] provided asymptotic capacity expressions and bounds for the MIMO IM/DD channel at high signal-to-noise ratio (SNR). Based on these results, we characterize the channel's outage probability diversity order (OPDO). This is the largest diversity order provided by this channel, i.e., no scheme, no matter how powerful, can achieve a larger diversity order. While the OPDA is an intrinsic channel property that can be achieved using information-theoretically optimal codes of

Y. Sapenov, A. Chaaban, and M.-S. Alouini are with the Computer, Electrical, and Mathematical Sciences and Engineering Division, King Abdullah University of Science and Technology (KAUST), Thuwal, Saudi Arabia. Email: \{yerzhan.sapenov,anas.chaaban,slim.alouini\}@kaust.edu.sa.

Z. Rezki is with the Department of Electrical and Computer Engineering, University of Idaho, Moscow, ID 83844-1023, USA. E-mail: zrezki@uidaho.edu.

M. Abdallah is with the College of Science and Engineering, Hamad Bin Khalifa University (HBKU), Qatar Foundation, Doha, Qatar. Email: moabdallah@hbku.edu.qa.

K. Qaraqe is with the Electrical Engineering Program, Texas A\&M Qatar, Doha, Qatar. Email: kqaraqe@tamu.edu.
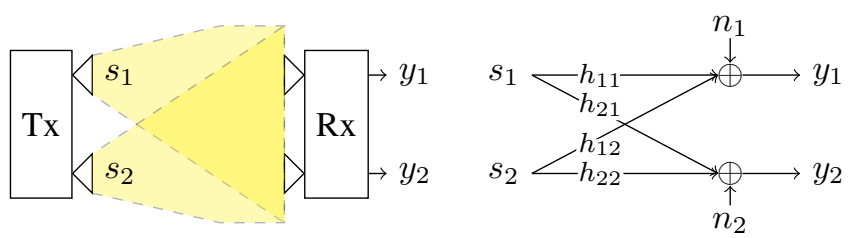

Fig. 1. A MIMO IM/DD system with $N_{t}=N_{r}=2: s_{i} \geq 0$ is the optical intensity of aperture $i, h_{i j} \geq 0$ is the channel gain from aperture $j$ to detector $i$, and $n_{i}$ is Gaussian noise.

large block length, it is practical to devise schemes that achieve the optimal diversity order with lower complexity. Here comes the role of the error probability diversity order (EPDO), which is a scheme property. We prove analytically that the EPDO of spatial RC coincides with the channels' OPDO, thus proving the diversity-order optimality of spatial RC for the MIMO IM/DD channel. Note that this is in sharp contrast with RF where diversity-optimality requires space-time coding. The result is applicable for any turbulence statistics of practical interest, as long as channels are independent and identically distributed (i.i.d.). The results are specialized to some practical turbulence statistics for comparison.

\section{SySTEM MODEL}

Consider an IM/DD OWC system with $N_{t}$ transmit and $N_{r}$ receive apertures as shown in Fig. 1. The transmission model in time instant $t$ can be represented as

$$
\mathbf{y}(t)=\mathbf{H}(t) \mathbf{s}(t)+\mathbf{n}(t),
$$

where $\mathbf{s}(t) \in \mathbb{R}_{+}^{N_{t} \times 1}$ is the transmit signal, $\mathbf{H}(t) \in \mathbb{R}_{+}^{N_{r} \times N_{t}}$ is the matrix of the channel coefficients, and $\mathbf{n}(t)$ is an $N_{r}$-dimensional vector of independent Gaussian noises with zero mean and variance $\sigma^{2}$. Noise is i.i.d. through time, and combines thermal noise and background radiation. The signal $\mathbf{s}(t)$ and the channel $\mathbf{H}(t)$ are nonnegative due to the use of IM/DD [5]. The channel model we consider in this paper is that of an IM/DD OWC system. Herein, the intensity of light source (laser, for instance) is modulated to send data to a receiver which detects using a photo-detector. This system is characterized by a nonnegative real transmit signal due to the use of intensity modulation (intensity is nonnegative), and positive channel coefficients due to direct detection (detected intensity is also nonnegative). Clearly, these constraints make the MIMO OWC and the MIMO RF channels fundamentally different. For instance, while Gaussian inputs are capacity-achieving for MIMO RF channels, Gaussian inputs are not even feasible in the MIMO OWC channel due to the non-negativity constraint of the intensity modulation. The positivity of the OWC channel coefficients is another fundamental feature that distinguishes $\mathrm{OWC}$ and $\mathrm{RF}$ 
TABLE I

DIVERSITY ORDER FOR IM/DD MIMO SYSTEMS.

\begin{tabular}{l|ccc}
\hline Channel statistics & Rayleigh & Exponential & $\operatorname{Gamma-Gamma}(\alpha, \beta)$ \\
\hline Diversity order & $2 N_{r} N_{t}$ & $N_{r} N_{t}$ & $N_{r} N_{t} \min (\alpha, \beta)$ \\
\hline
\end{tabular}

channels whose channel gains may be negative. As we show next, while it is well-known that coherent detection over a Rayleigh-distributed MIMO RF channel provides a maximum diversity gain equal to $N_{t} N_{r}$, the maximum diversity gain over a Rayleigh-distributed MIMO OWC channel is equal to $2 N_{t} N_{r}$. Throughout the paper, we assume the availability of channel state information (CSI) at the receiver, and that $\mathbf{H}(t)$ is quasi-static, i.e., $\mathbf{H}(k B+1)=\cdots=\mathbf{H}(k B+B)$ for $k=0,1, \ldots$ where $B$ is the block length. This models the very slow fading characteristic of OWC channels [7]. The components of $\mathbf{H}(t)$, denoted $h_{i j}(t)$, are i.i.d. according to a distribution $f_{h}(x), x \geq 0$, which can be realized if the transmit and receive apertures are properly spaced [5, p. 20]. The system has individual average optical intensity constraints $\mathbb{E}\left[s_{i}(t)\right] \leq \mathcal{E} \forall i \in\left\{1, \ldots, N_{t}\right\}$ and $\forall t$. We define SNR as $\gamma=\frac{\mathcal{E}}{\sigma} .{ }^{1}$ We study the channel's diversity order captured by the OPDO. We further study the EPDO achieved by RC with an $M$-PAM constellation. Those are defined respectively as

$$
d_{\mathrm{o}}=-\lim _{\gamma \rightarrow \infty} \frac{\log \mathbb{P}_{\mathrm{o}}\left(\gamma, R_{0}\right)}{\log \gamma}, \text { and } d_{\mathrm{e}}=-\lim _{\gamma \rightarrow \infty} \frac{\log \mathbb{P}_{\mathrm{e}}(\gamma, M)}{\log \gamma},
$$

where $\mathbb{P}_{\mathrm{o}}\left(\gamma, R_{0}\right)$ and $\mathbb{P}_{\mathrm{e}}(\gamma, M)$ are the channel outage probability at target rate $R_{0}$ and the error probability of $M$-PAM $\mathrm{RC}$ as a function of SNR $\gamma$, respectively.

\section{Main Results}

Let $l_{0}$ be the smallest integer such that $f_{h}^{\left(l_{0}\right)}(0) \neq 0$, where $f_{h}^{(k)}(a)$ denotes the $k^{\text {th }}$ derivative of $f_{h}(x)$ at $x=a$. The main results are as follows.

Theorem 1. The OPDO of the MIMO IM/DD channel is given by $d_{\mathrm{o}}=N_{r} N_{t}\left(l_{0}+1\right)$

Theorem 2. $R C$ achieves the maximum diversity order of the MIMO IM/DD channel, i.e., an EPDO of $d_{\mathrm{e}}=N_{r} N_{t}\left(l_{0}+1\right)$.

The proofs are given in Sections IV-A and IV-B, respectively. Since $d_{\mathrm{e}}=d_{\mathrm{o}}$, we conclude that $R C$ is diversity-optimal for the MIMO IM/DD channel.

Applying the results to some practical channel statistics leads to the results given in Table I. The Rayleigh distribution, $f_{h}(x)=\rho x e^{-\frac{\rho x^{2}}{2}}$ where $\rho>0$, models the impact of pointing errors caused by misalignment between the transmitter and receiver due to wind for instance [7]. In this case, $f_{h}^{(0)}(0)=0$ and $f_{h}^{(1)}(0) \neq 0$. Thus, $l_{0}=1$, and $d_{\mathrm{o}}=d_{\mathrm{e}}=2 N_{r} N_{t}$. The exponential and Gamma-Gamma distributions model strong and moderate/weak atmospheric turbulence caused by temperature variations along the propagation path, respectively [5, Ch. 11]. The exponential distribution, $f_{h}(x)=\eta e^{-\eta x}$ with $\eta>0$, satisfies $f_{h}^{(0)}(0) \neq 0$ and hence $l_{0}=0$ leading to $d_{\mathrm{o}}=d_{\mathrm{e}}=$ $N_{r} N_{t}$. The Gamma-Gamma distribution is given by $f_{h}(x)=$

\footnotetext{
${ }^{1}$ This is motivated by the high-SNR capacity of the SISO IM/DD channel given by $\log \left(\sqrt{\frac{e}{2 \pi}} \frac{\mathcal{E}}{\sigma}\right)$ [8].
}

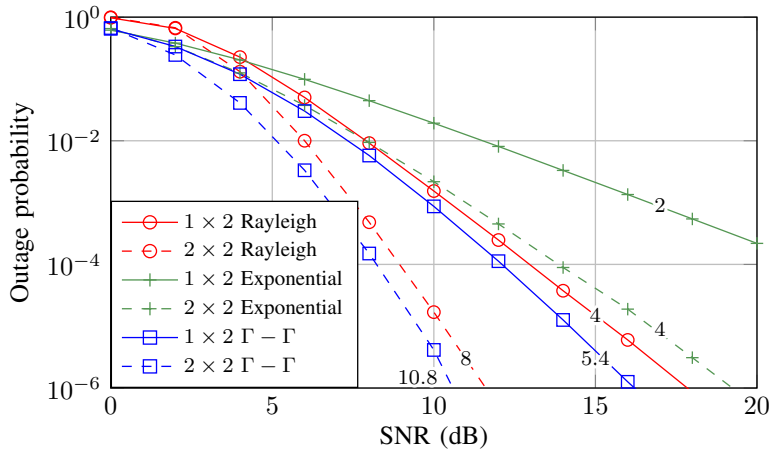

(a) Outage probability for $R_{0}=0.1$ nats.

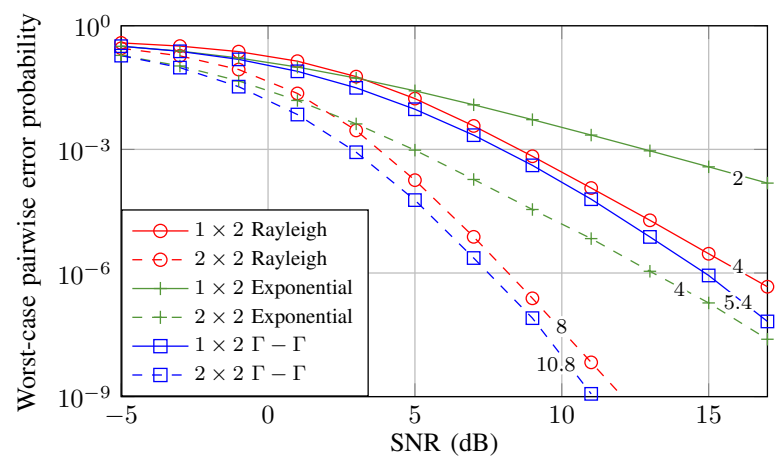

(b) Error probability for $M=2$

Fig. 2. Outage and error probability for an IM/DD $N_{t} \times N_{r}$ MIMO system as a function of $\operatorname{SNR}(\mathcal{E} / \sigma)$ under different channel turbulence statistics. The parameters are as follows: $\rho=0.5, \eta=1, \alpha=5.3$, and $\beta=2.7$. The markings on the plots show the diversity order.

$\frac{2(\alpha \beta)^{(\alpha+\beta) / 2}}{\Gamma(\alpha) \Gamma(\beta)} x^{(\alpha+\beta) / 2-1} K_{\alpha-\beta}(2 \sqrt{\alpha \beta x})$, where $\alpha$ and $\beta$ are positive parameters, and $K_{v}(u)$ is the modified Bessel function of the second kind. Using the alternative expression given by $f_{h}(x)=\sum_{j=0}^{\infty}\left(a_{j}(\alpha, \beta) x^{j+\beta-1}+a_{j}(\beta, \alpha) x^{j+\alpha-1}\right)$ [9], we get $l_{0}=\min (\alpha, \beta)-1$ and $d_{\mathrm{o}}=d_{\mathrm{e}}=N_{r} N_{t} \min (\alpha, \beta)$.

The results in this table agree with the literature on electrical-power-constrained MIMO IM/DD systems [9]-[11], and go beyond this point by proving the optimality of these diversity orders. Our result also provides a generic diversity order expression which applies for any analytic $f_{h}(x)$ on its support. Numerical evaluations are given in Fig. 2. The outage probability is plotted using (11), which is optimal at high SNR (cf. (4)). The worst-case PEP is plotted using (16). The numerical results agree with the derived statements on OPDO and EPDO. The proofs are given next.

\section{Derivation OF THE MAIN RESUlts}

In what follows, we drop the time index of $\mathbf{H}$, and treat it as a random matrix.

\section{A. Outage-Probability Diversity Order}

The outage probability can be defined as:

$$
\mathbb{P}_{\mathrm{o}}\left(\gamma, R_{0}\right)=\min _{p_{\mathbf{s}}} \operatorname{Pr}\left\{I(\mathbf{s} ; \mathbf{y} \mid \mathbf{H})<R_{0}\right\},
$$

where $R_{0}$ is the desired target rate, $p_{\mathbf{s}}$ is the distribution of $\mathbf{s}$ satisfying the intensity constraint, and $I(\mathbf{s} ; \mathbf{y} \mid \mathbf{H})$ is the mutual information between $\mathbf{s}$ and $\mathbf{y}$ given $\mathbf{H}$. To derive $d_{\mathrm{o}}$, we first bound $\mathbb{P}_{\mathrm{o}}\left(\gamma, R_{0}\right)$ as follows

$$
\begin{aligned}
\operatorname{Pr}\left\{I_{p_{\mathbf{s}}}(\mathbf{s} ; \mathbf{y} \mid \mathbf{H})<R_{0}\right\} & \geq \mathbb{P}_{\mathrm{o}}\left(\gamma, R_{0}\right) \\
& \geq \operatorname{Pr}\left\{\max _{p_{\mathbf{s} \mid \mathbf{H}}} I(\mathbf{s} ; \mathbf{y} \mid \mathbf{H})<R_{0}\right\}
\end{aligned}
$$


where $I_{p_{\mathbf{s}}}(\mathbf{s} ; \mathbf{y} \mid \mathbf{H})$ is the mutual information when $\mathbf{s}$ follows a distribution $p_{\mathbf{s}}$ independent of $\mathbf{H}$. Next, we focus on the case $N_{t} \leq N_{r}$. The other case is relegated to the appendix. Note that $\max _{p_{\mathbf{s} \mid \mathbf{H}}} I(\mathbf{s} ; \mathbf{y} \mid \mathbf{H})$ is the channel capacity as a function of $\gamma$ and $\mathbf{H}$. This in turn is upper bounded by the channel capacity under a sum intensity constraint, which is upper bounded by $\frac{1}{2} \log \left|\frac{e \gamma^{2}}{2 \pi} \mathbf{H}^{T} \mathbf{H}\right|+\epsilon_{\gamma}$, where $\lim _{\gamma \rightarrow \infty} \frac{\epsilon_{\gamma}}{\log (\gamma)}=0$ [6]. Thus,

$$
\begin{aligned}
\mathbb{P}_{\mathrm{o}}\left(\gamma, R_{0}\right) & \geq \operatorname{Pr}\left\{\frac{1}{2} \log \operatorname{det}\left|\frac{e \gamma^{2}}{2 \pi} \mathbf{H}^{T} \mathbf{H}\right|<R_{0}-\epsilon_{\gamma}\right\} \\
& =\operatorname{Pr}\left\{\sum_{j=1}^{N_{t}} \log \left(\frac{e \gamma^{2}}{2 \pi}\left\|\mathbf{h}_{j}\right\|_{2}^{2}\right)<2\left(R_{0}-\epsilon_{\gamma}\right)\right\},
\end{aligned}
$$

where we used $\log |\mathbf{A}|=\operatorname{tr}(\log (\mathbf{A})$ ) (componentwise $\log$ ) for real $\mathbf{A}$, and where $\mathbf{h}_{j}$ is $j^{\text {th }}$ column of $\mathbf{H}$. Since

$$
\operatorname{Pr}\left\{\sum_{i=1}^{N} a_{i}<b\right\} \geq \prod_{i=1}^{N} \operatorname{Pr}\left\{a_{i}<\frac{b}{N}\right\}
$$

for mutually independent $a_{1}, \ldots, a_{N}$, we obtain

$$
\begin{aligned}
\mathbb{P}_{\mathrm{o}}\left(\gamma, R_{0}\right) & \geq \prod_{j=1}^{N_{t}} \operatorname{Pr}\left\{\log \left(\frac{e \gamma^{2}}{2 \pi}\left\|\mathbf{h}_{j}\right\|_{2}^{2}\right)<\frac{2\left(R_{0}-\epsilon_{\gamma}\right)}{N_{t}}\right\} \\
& =\prod_{j=1}^{N_{t}} \operatorname{Pr}\left\{\left\|\mathbf{h}_{j}\right\|_{2}^{2}<\frac{2 \pi}{\gamma^{2}} e^{\frac{2\left(R_{0}-\epsilon \gamma\right)}{N_{t}}-1}\right\} \\
& \geq \prod_{j=1}^{N_{t}} \prod_{i=1}^{N_{r}} \operatorname{Pr}\left\{h_{i j}<\frac{k_{1}}{\gamma}\right\},
\end{aligned}
$$

using (5) and since $h_{i j} \geq 0$, where $k_{1}=\frac{\sqrt{2 \pi}}{\sqrt{N_{r}}} e^{\frac{2\left(R_{0}-\epsilon \gamma\right)-N_{t}}{2 N_{t}}}$. Due to the independence of $h_{i j}$, this can be written as

$$
\mathbb{P}_{\mathrm{o}}\left(\gamma, R_{0}\right) \geq\left[F_{h}\left(\frac{k_{1}}{\gamma}\right)\right]^{N_{t} N_{r}}
$$

where $F_{h}(x)$ is the CDF of $h$. Using the Taylor series expansion of $f_{h}(h)$ at $h=0$, we obtain

$$
\begin{aligned}
F_{h}\left(\frac{k_{1}}{\gamma}\right) & =\int_{0}^{\frac{k_{1}}{\gamma}} f_{h}(h) d h=\int_{0}^{\frac{k_{1}}{\gamma}} \sum_{n=l_{0}}^{\infty} \frac{f_{h}^{(n)}(0)}{n !} h^{n} d h \\
& =c\left(l_{0}\right) \gamma^{-l_{0}-1}+c\left(l_{0}+1\right) \gamma^{-l_{0}-2}+\cdots
\end{aligned}
$$

where $f_{h}^{(n)}(h)$ is the $n^{\text {th }}$ derivative of $f_{h}(h), c(l)=\frac{f_{h}^{(l)}(0) k_{1}^{l+1}}{(l+1) !}$ and the second inequality in (9) follows since $f_{h}(x)$ is analytic on its support. Substituting in (8), taking the logarithm, dividing by $\log (\gamma)$, and taking the limit as $\gamma \rightarrow \infty$ yields $d_{\mathrm{o}} \leq N_{r} N_{t}\left(l_{0}+1\right)$ if $N_{t} \leq N_{r}$.

To derive a lower bound, we fix $p_{\mathbf{s}}$ in (3) to be i.i.d. exponential with mean $\mathcal{E}$. Substituting in the left-hand side of (3), and using the entropy power inequality to lower bound the mutual information as in [4, (30)], yields

$$
\mathbb{P}_{\mathrm{o}}\left(\gamma, R_{0}\right) \leq \operatorname{Pr}\left\{\frac{1}{2} \log \left|\frac{e \gamma^{2}}{2 \pi} \mathbf{H}^{T} \mathbf{H}\right|<R_{0}\right\}
$$

Now we use $\log |\mathbf{A}|=\operatorname{tr}(\log (\mathbf{A}))$, followed by the inequality $\operatorname{Pr}\left\{\sum_{i=1}^{N} a_{i}<b\right\} \leq \prod_{i=1}^{N} \operatorname{Pr}\left\{a_{i}<b\right\}$ which holds for mutually independent $a_{1}, \ldots, a_{N}$, to obtain

$$
\begin{aligned}
\mathbb{P}_{\mathrm{o}}\left(\gamma, R_{0}\right) & \leq \prod_{j=1}^{N_{t}} \operatorname{Pr}\left\{\left\|\mathbf{h}_{j}\right\|_{2}^{2}<\frac{2 \pi}{\gamma^{2}} e^{2 R_{0}-1}\right\} \\
& \leq \prod_{j=1}^{N_{t}} \prod_{i=1}^{N_{r}} \operatorname{Pr}\left\{h_{i j}<\frac{k_{2}}{\gamma}\right\},
\end{aligned}
$$

where $k_{2}=\sqrt{2 \pi} e^{\frac{2 R_{0}-1}{2}}$. Thus,

$$
\mathbb{P}_{\mathrm{o}}\left(\gamma, R_{0}\right) \leq\left[F_{h}\left(\frac{k_{2}}{\gamma}\right)\right]^{N_{t} N_{r}} .
$$

Using (10) leads to the lower bound $d_{\mathrm{o}} \geq N_{r} N_{t}\left(l_{0}+1\right)$, which coincides with the upper bound, thus proving the OPDO statement in Theorem 1 for $N_{t} \leq N_{r}$. The same holds for the case $N_{t}>N_{r}$ as shown in the appendix.

\section{B. Error Probability Diversity Order of $R C$}

The error probability of $M$-PAM RC, $\mathbb{P}_{\mathrm{e}}(\gamma, M)$, can be bounded by using the worst-case PEP, $\mathbb{P}_{\mathrm{e}}^{[\mathrm{o}]}(\gamma, M)$, as [12]:

$$
\frac{N_{\min }}{M} \mathbb{P}_{\mathrm{e}}^{[\mathrm{o}]}(\gamma, M) \leq \mathbb{P}_{\mathrm{e}}(\gamma, M) \leq(M-1) \mathbb{P}_{\mathrm{e}}^{[\mathrm{o}]}(\gamma, M),
$$

where $N_{\min }$ is the number of symbols that are at the minimum distance from at least one other symbol. From [13], we know that $\mathbb{P}_{\mathrm{e}}^{[\mathrm{o}]}(\gamma, M)$ has following form under individual optical intensity constraint and using $\mathrm{RC}$ :

$$
\mathbb{P}_{\mathrm{e}}^{[\mathrm{o}]}(\gamma, M)=\mathbb{E}_{\mathbf{H}}\left[Q\left(\frac{\gamma}{(M-1)}\left\|\mathbf{H} \mathbf{1}_{N_{t} \times 1}\right\|_{2}\right)\right],
$$

where $Q(x)$ is the $Q$-function, $\mathbf{1}_{N_{t} \times 1}$ is an all-ones $N_{t} \times 1$ vector, and $\mathbb{E}_{\mathbf{H}}[\cdot]$ is the expectation with respect to $\mathbf{H}$. We can upper and lower bound this using the following:

$$
\sqrt{\frac{e}{2 \pi}} \frac{\sqrt{\beta-1}}{\beta} e^{-\frac{\beta x^{2}}{2}} \leq Q(x) \leq e^{-\frac{x^{2}}{2}}, \text { for } x>0,
$$

where $\beta$ is an arbitrary positive real number [14]. Thus,

$$
\mathbb{P}_{\mathrm{e}}^{[\mathrm{o}]}(\gamma, M) \leq \mathbb{E}_{\mathbf{H}}\left[e^{-\frac{\gamma^{2}}{2(M-1)^{2}}\left\|\mathbf{H} \mathbf{1}_{N_{t} \times 1}\right\|_{2}^{2}}\right]
$$

Note that $\left\|\mathbf{H} \mathbf{1}_{N_{t} \times 1}\right\|_{2}^{2} \geq \sum_{i=1}^{N_{r}} \sum_{j=1}^{N_{t}} h_{i j}^{2}\left(h_{i j} \geq 0\right)$. Using this and the independence of $h_{i j}$ yields

$$
\begin{aligned}
\mathbb{P}_{\mathrm{e}}^{[\mathrm{o}]}(\gamma, M) & \leq \mathbb{E}_{\mathbf{H}}\left[e^{-s \sum_{i=1}^{N_{r}} \sum_{j=1}^{N_{t}} h_{i j}^{2}}\right] \\
& =\prod_{i=1}^{N_{r}} \prod_{j=1}^{N_{t}} \mathbb{E}_{\mathbf{H}}\left[e^{-s h_{i j}^{2}}\right]=\left[\mathbb{E}_{h}\left[e^{-s h^{2}}\right]\right]^{N_{r} N_{t}},
\end{aligned}
$$

where $s=\frac{\gamma^{2}}{2(M-1)^{2}}$. Therefore, $\mathbb{P}_{\mathrm{e}}^{[\mathrm{o}]}(\gamma, M) \leq$ $\left[\int_{0}^{\infty} e^{-s h^{2}} f_{h}(h) d h\right]^{N_{r} N_{t}}$. Using the Taylor series expansion of $f_{h}(h)$ at $h=0$, we get

$$
\begin{aligned}
& \mathbb{P}_{\mathrm{e}}^{[\mathrm{o}]}(\gamma, M) \leq\left[\int_{0}^{\infty} e^{-s h^{2}} \sum_{n=l_{0}}^{\infty} \frac{f_{h}^{(n)}(0)}{n !} h^{n} d h\right]^{N_{r} N_{t}} \\
& =\left[d\left(l_{0}\right) s^{-\frac{l_{0}+1}{2}}+d\left(l_{0}+1\right) s^{-\frac{l_{0}+2}{2}}+\cdots\right]^{N_{r} N_{t}}
\end{aligned}
$$


where $d(l)=\frac{f_{h}^{(l)}(0)}{l !} \frac{\Gamma\left(\frac{l+1}{2}\right)}{2}$ and $\Gamma(\cdot)$ is the Gamma function. At high SNR $(\gamma \rightarrow \infty)$, the first term dominates in (21). Taking the logarithm of (21), dividing by $\log (\gamma)$, and letting $\gamma \rightarrow \infty$ leads to the EPDO lower bound $d_{\mathrm{e}} \geq N_{r} N_{t}\left(l_{0}+1\right)$.

Using the lower bound in (17), and the inequality $\left\|\mathbf{H} 1_{N_{t} \times 1}\right\|_{2}^{2} \leq N_{t} \sum_{i=1}^{N_{r}} \sum_{j=1}^{N_{t}} h_{i j}^{2}$, and proceeding with similar steps as (19)-(21), we can lower bound (16) as $\mathbb{P}_{\mathrm{e}}^{[\mathrm{o}]}(\gamma, M) \geq\left[\int_{0}^{\infty} K e^{-\tilde{s} N_{t} h^{2}} f(h) d h\right]^{N_{r} N_{t}}$, where $\tilde{s}=$ $\frac{\beta \gamma^{2}}{2(M-1)^{2}}$ and $K=\sqrt{\frac{e}{2 \pi}} \frac{\sqrt{\beta-1}}{\beta}$. Using the Taylor series expansion of $f_{h}(h)$ leads to $d_{\mathrm{e}} \leq N_{r} N_{t}\left(l_{0}+1\right)$. Hence, the upper and lower bounds coincide, proving $d_{\mathrm{e}}$ in Theorem 2 .

\section{CONClusion}

The outage diversity represents the maximum possible diversity achievable in the system, it represents the physical capability of the channels at high SNR. In this paper, we characterized such a diversity and proved that the error probability diversity of RC is equal to the outage diversity, the maximum possible. Our results hold true irrespective of the optical channel statistics. Specializing our results to most popular turbulence models, we have explicitly derived their diversity orders, thus capturing several independent results.

\section{ACKNOWLEDGMENT}

This publication was made possible by NPRP grant number 9-077-2-036 from the Qatar National Research Fund (a member of Qatar Foundation). The statements made herein are solely the responsibility of the authors.

\section{APPENDIX}

To derive the OPDO for $N_{t}>N_{r}$, we consider the capacity upper bound given in $[6,(91)]$ as $C_{s}(\mathbf{H}, \gamma) \leq$ $\sum_{i=1}^{N_{r}} \frac{1}{2} \log \left(\frac{e N_{t}^{2} \gamma^{2}}{2 \pi}\left\|\mathbf{h}_{i}\right\|_{1}^{2}\right)+\epsilon_{\gamma}$. Using this bound, and following similar steps as (4)-(8) leads to $d_{\mathrm{o}} \leq N_{r} N_{t}\left(l_{0}+1\right)$.

To derive a lower bound by reusing (11), we transform the channel into an effective $N_{r} \times N_{r}$ channel as follows. We choose $\mathbf{s}=\mathbf{G x}$, where $\mathbf{G}$ is an $N_{t} \times N_{r}$ matrix and $\mathbf{x}$ is i.i.d. exponential with $N_{r}$ components, each with mean $\mathcal{E}$. To harness the whole diversity of the channel, we choose $\mathbf{G}=\left[\begin{array}{cc}\mathbf{I}_{N_{r}-1} & \mathbf{0}_{N_{r}-1 \times 1} \\ \mathbf{0}_{\left(N_{t}-N_{r}+1\right) \times\left(N_{r}-1\right)} & \mathbf{1}_{\left(N_{t}-N_{r}+1\right) \times 1}\end{array}\right]$, where $\mathbf{I}_{a}$ is an $a \times a$ identity matrix, and $\mathbf{0}_{a \times b}$ is an $a \times b$ all zeros matrix. Then, transmitting $\mathbf{s}$ is equivalent to transmitting $\mathbf{x}$ over a channel with an effective channel matrix $\mathbf{H}_{\mathrm{e}}=\mathbf{H G}$. Writing $\mathbf{H}=\left[\mathbf{H}_{1}, \mathbf{H}_{2}\right]$ where $\mathbf{H}_{1}$ is the first $N_{t}-1$ columns of $\mathbf{H}$, and $\mathbf{H}_{2}$ the rest, leads to $\mathbf{H}_{\mathrm{e}}=\left[\mathbf{H}_{1}, \mathbf{H}_{2} \mathbf{1}_{\left(N_{t}-N_{r}+1\right) \times 1}\right]$. Then, applying (11) we obtain

$$
\mathbb{P}_{\mathrm{o}}\left(\gamma, R_{0}\right) \leq \operatorname{Pr}\left\{\frac{1}{2} \log \left|\frac{e \gamma^{2}}{2 \pi} \mathbf{H}_{e}^{T} \mathbf{H}_{e}\right|<R_{0}\right\} .
$$

Using $\log |\mathbf{A}|=\operatorname{tr}(\log (\mathbf{A}))$, we can write $\mathbb{P}_{\mathrm{o}}\left(\gamma, R_{0}\right) \leq$ $\operatorname{Pr}\left\{A_{1}+A_{2}<R_{0}\right\}$ where

$$
\begin{aligned}
& A_{1}=\frac{1}{2} \sum_{i=1}^{N_{r}-1} \log \left(\frac{e \gamma^{2}}{2 \pi}\left\|\mathbf{h}_{1, i}\right\|_{2}^{2}\right), \\
& A_{2}=\frac{1}{2} \log \left(\frac{e \gamma^{2}}{2 \pi}\left\|\mathbf{H}_{2} \mathbf{1}_{\left(N_{t}-N_{r}+1\right) \times 1}\right\|_{2}^{2}\right),
\end{aligned}
$$

and $\mathbf{h}_{1, i}$ is the $i^{\text {th }}$ column of $\mathbf{H}_{1}$. Thus $\mathbb{P}_{\mathrm{o}}\left(\gamma, R_{0}\right) \leq$ $\operatorname{Pr}\left\{A_{1}<R_{0}\right\} \operatorname{Pr}\left\{A_{2}<R_{0}\right\}$. Using similar steps as (11)(14) yields $\operatorname{Pr}\left\{A_{1}<R_{0}\right\} \leq\left[F_{h}\left(\frac{k_{2}}{\gamma}\right)\right]^{N_{r}\left(N_{r}-1\right)}$ where $k_{2}=$ $\sqrt{2 \pi} e^{\frac{2 R_{0}-1}{2}}$. Moreover,

$$
\begin{aligned}
\operatorname{Pr}\left\{A_{2}<R_{0}\right\} & =\operatorname{Pr}\left\{\left\|\mathbf{H}_{2} \mathbf{1}_{\left(N_{t}-N_{r}+1\right) \times 1}\right\|_{2}^{2}<\frac{2 \pi}{\gamma^{2}} e^{2 R_{0}-1}\right\} \\
& \leq \prod_{i=1}^{N_{r}} \operatorname{Pr}\left\{\left\|\mathbf{h}_{2, i}\right\|_{1}^{2}<\frac{2 \pi}{\gamma^{2}} e^{2 R_{0}-1}\right\} \\
& \leq \prod_{i=1}^{N_{r}} \prod_{j=1}^{N_{t}-N_{r}+1} \operatorname{Pr}\left\{h_{2, i j}<\frac{\sqrt{2 \pi}}{\gamma} e^{\frac{2 R_{0}-1}{2}}\right\},
\end{aligned}
$$

where $\mathbf{h}_{2, i}$ is the $i^{\text {th }}$ row of $\mathbf{H}_{2}$, and $h_{2, i j}$ is the $j^{\text {th }}$ element of $\mathbf{h}_{2, i}$. Thus $\operatorname{Pr}\left\{A_{2}<R_{0}\right\} \leq\left[F_{h}\left(\frac{k_{2}}{\gamma}\right)\right]^{N_{r}\left(N_{t}-N_{r}+1\right)}$. Combining terms yields $\mathbb{P}_{\mathrm{o}}\left(\gamma, R_{0}\right) \leq\left[F_{h}\left(\frac{k_{2}}{\gamma}\right)\right]^{N_{r} N_{t}}$ which by using (10) leads to $d_{\mathrm{o}} \geq N_{r} N_{t}\left(l_{0}+1\right)$. The bounds coincide, thus proving Theorem 1 for the case $N_{t}>N_{r}$.

\section{REFERENCES}

[1] M. A. Khalighi and M. Uysal, "Survey on free space optical communications: A communication theory perspective," Commun. Surveys Tuts., vol. 16, no. 4, pp. 2231-2258, 4th quarter 2014.

[2] T. Fath and H. Haas, "Performance Comparison of MIMO Techniques for Optical Wireless Communications in Indoor Environments," IEEE Trans. Commun., vol. 61, no. 2, pp. 733-742, February 2013.

[3] A. Chaaban, Z. Rezki, and M.-S. Alouini, "MIMO intensity-modulation channels: Capacity bounds and high SNR characterization," in Proc. IEEE Int. Conf. Commun. (ICC), Paris, France, May 2017.

[4] S. M. Moser, M. Mylonakis, L. Wang, and M. Wigger, "Asymptotic capacity results for MIMO wireless optical communication," in Proc. IEEE Int. Symp. Inf. Theory (ISIT), Aachen, Germany, Jun. 2017.

[5] S. Arnon, J. Barry, G. Karagiannidis, R. Schober, and M. Uysal, Eds., Advanced Optical Wireless Communication Systems. Cambridge University Press, 2012.

[6] A. Chaaban, Z. Rezki, and M.-S. Alouini, "Capacity Bounds and High-SNR Capacity of MIMO Intensity-Modulation Optical Channels," KAUST Technical Report http://hdl.handle.net/10754/624895, June 2017.

[7] A. A. Farid and S. Hranilovic, "Outage capacity optimization for freespace optical links with pointing errors," J. Lightw. Technol., vol. 25, no. 7, pp. 1702-1710, Jul. 2007.

[8] A. Lapidoth, S. M. Moser, and M. Wigger, "On the capacity of freespace optical intensity channels," IEEE Trans. Inf. Theory, vol. 55, no. 10, pp. 4449-4461, Oct. 2009.

[9] E. Bayaki, R. Schober, and R. K. Mallik, "Performance analysis of MIMO free-space optical systems in gamma-gamma fading," IEEE Trans. Commun., vol. 57, no. 11, pp. 3415-3424, Nov 2009.

[10] S. G. Wilson, M. Brandt-Pearce, Q. Cao, and M. Baedke, "Optical repetition MIMO transmission with multipulse PPM," IEEE J. Sel. Areas Commun., vol. 23, no. 9, pp. 1901-1910, Sept 2005.

[11] M. Safari and M. Uysal, "Cooperative diversity over log-normal fading channels: performance analysis and optimization," IEEE Trans. Wireless Commun., vol. 7, no. 5, pp. 1963-1972, May 2008.

[12] M. Salehi and J. Proakis, Digital Communications. McGraw-Hill Education, 2007.

[13] Y. Sapenov, A. Chaaban, Z. Rezki, and M.-S. Alouini, "On the Optimality of Repetition Coding among Rate-1 DC-offset STBCs for MIMO Optical Wireless Communications," KAUST Technical Report http://hdl.handle.net/10754/624894, June 2017.

[14] S. H. Chang, P. C. Cosman, and L. B. Milstein, "Chernoff-Type Bounds for the Gaussian Error Function," IEEE Trans. Commun., vol. 59, no. 11, pp. 2939-2944, November 2011. 